\title{
Primeiro Registro de Ectopsocus titschacki Jentsch (Psocodea: Psocoptera: Ectopsocidae) para o Estado da Bahia: Uma Prova da Falta de Estudos nessa Ordem de Insecta no Brasil*
}

\author{
Alberto Moreira Silva-Neto ${ }^{\bowtie}$, Freddy Bravo $^{1}$ \& Alfonso N. García Aldrete ${ }^{2}$
}

1. Universidade Estadual de Feira de Santana, e-mail: bio.alberto@gmail.com (Autor para correspondência ${ }^{\bowtie}$ ), freddy11bravo@yahoo.com.br. 2. Universidad Nacional Autónoma do México, e-mail: anga@ibunam2.ibiologia.unam.mx.

\section{EntomoBrasilis 6 (1): 86-88 (2013)}

Resumo. Nesse trabalho realizamos o primeiro registro de Ectopsocus titschacki Jentsch para o Estado da Bahia, incluindo alguns comentários sobre a atual situação do conhecimento e da distribuição dessa ordem de Insecta no Brasil evidenciando a lacuna de conhecimento desse grupo em alguns estados especialmente aqueles pertencentes à região Nordeste.

Palavas-Chave: Distribuição; Neotrópico; Psocídeos.

\section{First Record of Ectopsocus titschacki Jentsch (Psocodea: Psocoptera: Ectopsocidae) in Bahia State: Proof of a Lack of Studies on this Order of Insecta in Brazil}

Abstract. In this paper we report the first record of Ectopsocus titschacki Jentsch for the State of Bahia, including some comments on the current state of knowledge and distribution of this order of Insecta in Brazil showing the knowledge gap that group in some states especially those belonging to Northeast.

Keywords: Distribution; Neotropics; Psocids.

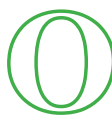
s psocópteros são insetos de ampla distribuição mundial; ao final de 2005 se conhecia 5557 espécies, em 474 gêneros e 41 famílias (GARCía AldRete 2006). O catálogo de Psocoptera (Lienhard \& SMithers 2002) lista para o Brasil 374 espécies em 87 gêneros e 29 famílias. Recentemente, GARCía ALDRETE \& MOCKFORD (2009) atualizaram a lista de psocópteros do Brasil para 425 espécies em 94 gêneros e 28 famílias, após análise de espécimes da coleção de Invertebrados do Instituto Nacional de Pesquisas da Amazônia, Manaus, Amazonas Brasil (INPA) e da Coleção Entomológica Professor Johann Becker do Museu de Zoologia da Universidade Estadual de Feira de Santana, Feira de Santana, Bahia, Brasil (MZFS)

O gênero Ectopsocus possui 156 espécies distribuídas em várias partes do mundo e pertencente à família Ectopsocidae. (LIENHARD $\&$ SMithers 2002). Cinco espécies desse gênero ocorrem no Brasil: Ectopsocus pumilis (Banks), com registro para o estado de São Paulo; Ectopsocus richardsi (Pearman), com ampla distribuição no Brasil; Ectopsocus ribagai Enderlein, Ectopsocus titschacki Jentsch e Ectopsocus vilhenai Badonnel, todas com registro no estado do Pará (García Aldrete \& Mockford 2009).

Os machos de Ectopsocus possuem uma fileira de dentículos ao longo da margem posterior do clunium antes do epiprocto; as fêmeas do gênero possuem valvas ovipositoras completas (v1, v2 e v3) e placa subgenital ligeiramente bilobada posteriormente (MOCKFORD 1993).

O objetivo deste trabalho foi registrar pela primeira vez a ocorrência de E. titschacki para o estado da Bahia.
Foi realizada uma coleta no período de 06 á 11 de novembro de 2010 em um fragmento de Mata Atlântica, Serra da Jibóia, município de Santa Terezinha $\left(12^{\circ} 51^{\prime} \mathrm{S}\right.$ e $39^{\circ} 28^{\prime}$ O) com 6 km de extensão, com trechos de caatinga na base, mata higrófila nas encostas e um afloramento rochoso de origem gnásico-granítica no topo (VAlente \& PôRTO 2006); a Serra da Jibóia é uma das 147 áreas prioritárias para conservação do Bioma Mata Atlântica (BRASIL 2002).

A coleta foi realizada com guarda chuva entomológico. Um exemplar macho adulto de E. titschacki (Figura 2) foi coletado e mantido em álcool 70\% até a realização da determinação, segundo a classificação de SMithers (1990) e confirmação por um de nós (ANGA). Foram realizadas cinco coletas anteriores na mesma área, porém apenas com armadilhas luminosas (tipo Luiz de Queiroz) que talvez tenha pouca eficiência na coleta de algumas espécies de Psocoptera que não sejam atraídas por luz. Esse talvez seja o caso de E. titschacki.

A espécie E. titschacki é amplamente distribuída com registro de ocorrência em várias partes do mundo: Alemanha, Espanha, Estados Unidos da América, Antilhas, Costa Rica, Cuba, Guatemala, México, Panamá, Guiana Francesa, Venezuela, Brasil, Angola, Congo, Guiné Equatorial, Nigéria, Senegal e Indonésia (MOCKFORD 1993).

*Este trabalho foi financiado pelo Ministério de Ciência e Tecnologia (PPBIO Semi-árido). 
T No Brasil, porém, como citado anteriormente, só existe registro de E. titschacki no Pará (próximo ao equador geográfico), e com o registro apresentado neste trabalho na Bahia (aproximadamente $13^{\circ} \mathrm{S}$ ), demonstra o abismo existente com relação ao conhecimento de Psocoptera no país, pois mesmo tratando-se de uma espécie amplamente distribuída no planeta, sua ocorrência no Brasil foi relatada apenas em um dos 26 estados brasileiros.

Antes da atualização do catálogo de Psocoptera de LiEnhard e Smithers (2002) realizada na lista de espécies do Brasil por GARCíA ALDRETE \& MoCKFoRd (2009), na região Nordeste do Brasil existia o registro de apenas doze espécies, sendo sete ocorrências no estado de Pernambuco e cinco na Bahia, sem nenhum registro para a região Semi-árida.

Após a atualização de GARCía Aldrete \& Mockford (2009), o número de registros de Psocoptera na Bahia triplicou, passando de cinco para 15 espécies (Figura 1). Antes dessa atualização, segundo o catálogo de Lienhard e SMithers (2002), as cinco espécies registradas para a Bahia estavam distribuídas em duas famílias: 1) Dolabellopsocidae, com três espécies: Dolabellopsocus ctenatus (New), Dolabellopsocus pectenatus Eertmoed (endêmico) e Isthmopsocus radulatus New (endêmico); 2) Lachesillidae, com duas espécies: Lachesilla bahiana García Aldrete (endêmica) e Lachesilla convexa García Aldrete. GARCíA ALDRETE \& MOCKFORD (2009) acrescentaram mais uma subordem e quatro famílias: a subordem Trogiomorpha, com duas espécies da família Lepidopsocidae (Echmepteryx falco (Badonnel), com registro anterior apenas para o Amazonas e Echmepteryx madagascariensis (Kolbe), com registro anterior apenas para o Rio de Janeiro, e três novas famílias da subordem Psocomorpha, sendo Epipsocidae, com três espécies, sendo uma delas com o primeiro registro para a região semi-árida, [Gênero ca. Goja. Bahia (endêmica), Mesepipsocus sp. 1. Bahia (Senhor do Bonfim e Ituberá)] (endêmica) e Mesepipsocus sp. 2. Bahia. (endêmica). A família Ptiloneuridae com três espécies, sendo uma delas com ocorrência no Semi-árido (Euplocania picta sp. 1. Bahia (endêmica), Triplocania ariasi New, antes com registro apenas para o Amazonas e Triplocania sp. 1. Bahia (Senhor do Bonfim e Santa Terezinha) ( endêmica), e por último a família Caeciliusidae, com uma espécie (Stenocaecilius casarum (Badonnel) que possui ampla distribuição nos trópicos, mas que somente após essa atualização foi registrada no Brasil.

Considerando as 425 espécies de Psocoptera registradas no Brasil, García Aldrete \& Mockford (2009) consideram 312 delas como endêmicas de algum estado ou região do Brasil. Estes autores discutem depois, esta alta taxa de endemismo de espécies pode estar relacionada com a falta de estudo e trabalhos com este grupo de insetos no nosso país.

Na medida em que os estudos com psocópteros nos estados brasileiros aumentar de forma equilibrada entre as Regiões, a tendência é que os casos de endemismo por estados venham a ter uma diminuição considerável, demonstrando de forma mais clara e real os padrões de distribuição das espécies de Psocoptera no Brasil.

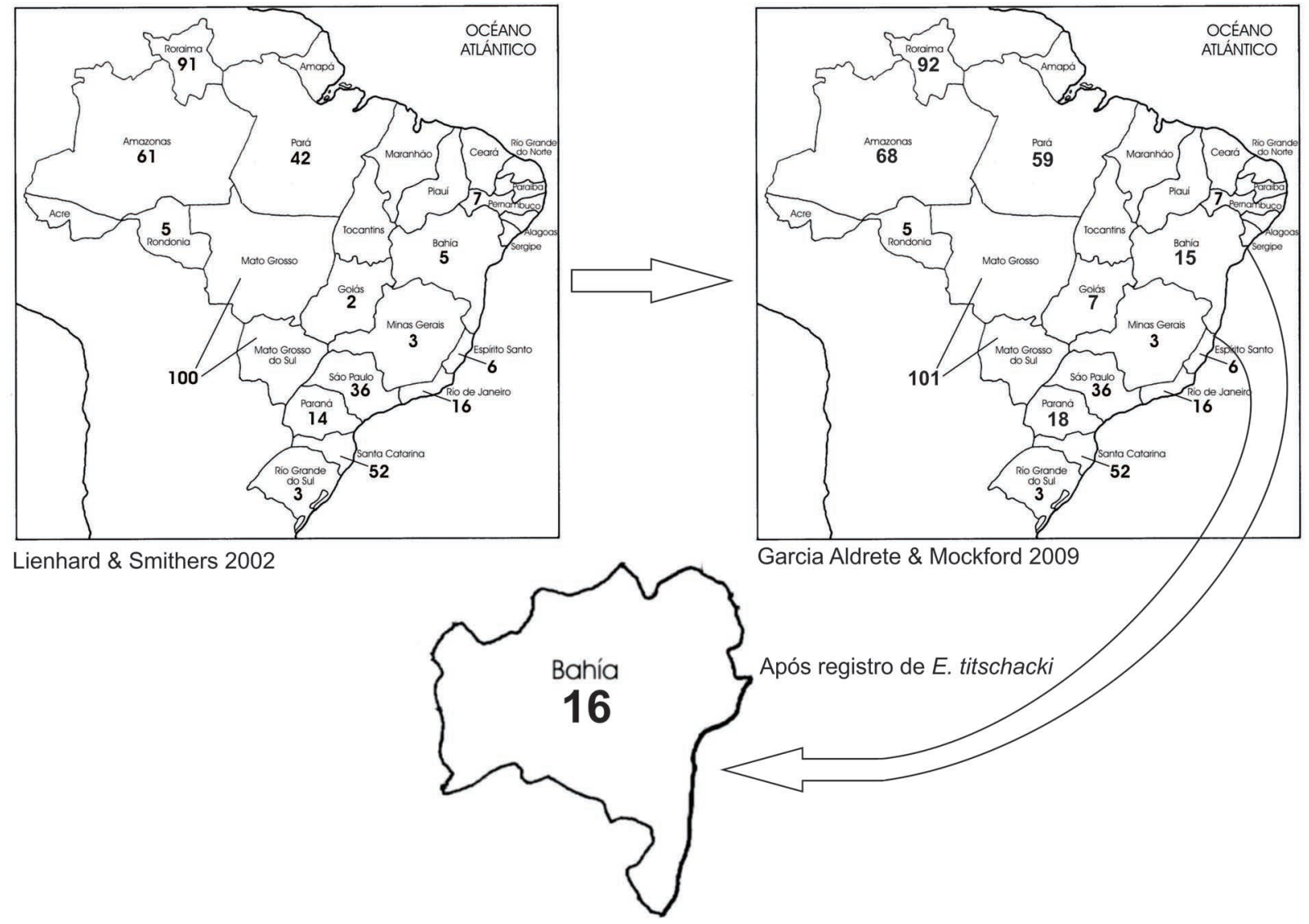

Figura 1. Distribuição dos Psocoptera pelos Estados do Brasil antes da atualização de GARCía ALDRETE \& MockFord (2009), depois da atualização e o número de espécies presentes na Bahia após o registro de $E$. titschacki. 

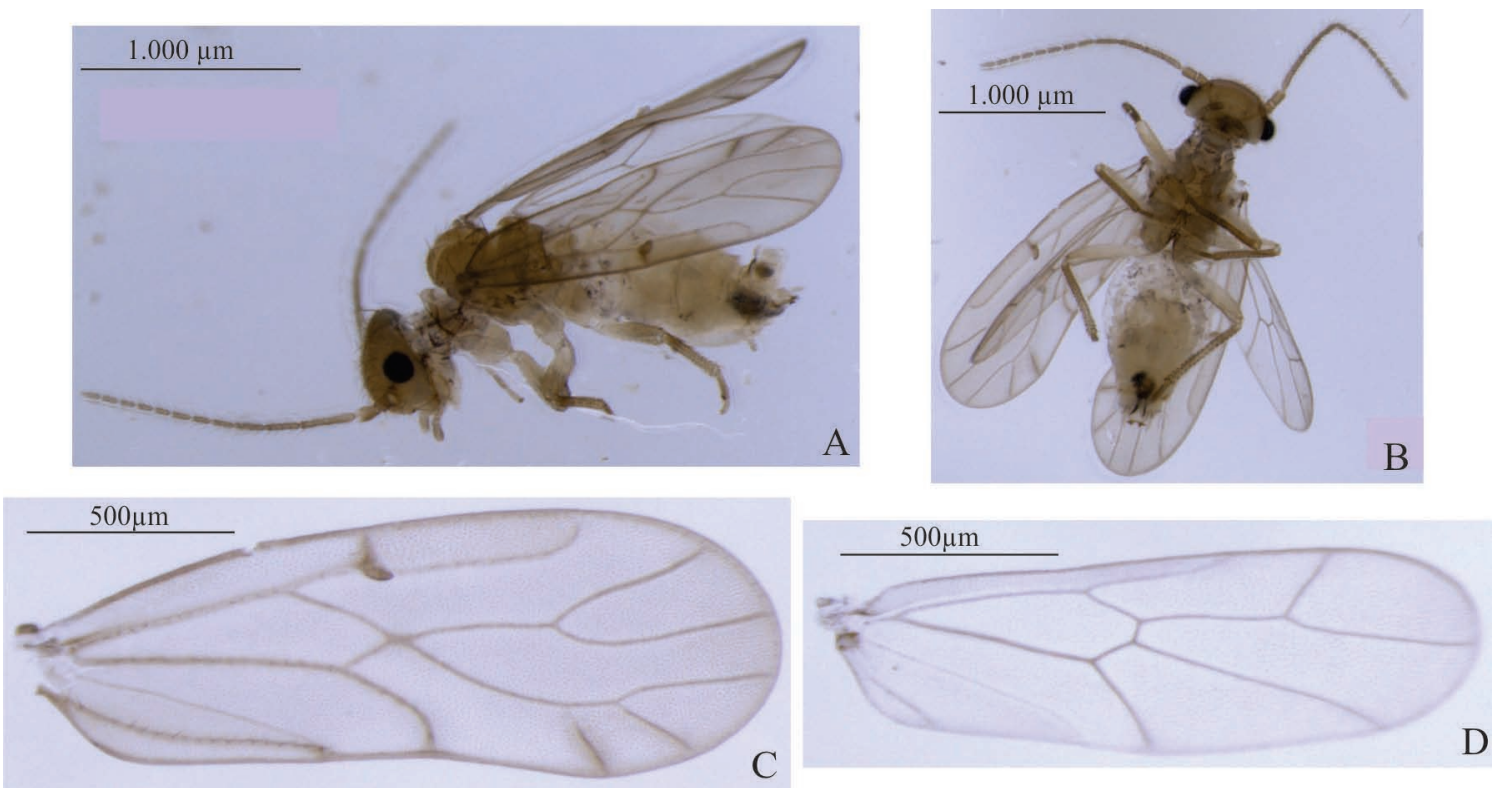

Figura 2. Espécime coletado de E. titschaki. A: Vista lateral. B: Vista ventral. C: Asa anterior. D: Asa posterior.

\section{AGRADECIMENTOS}

Agradecemos ao Projeto de Pesquisa em Biodiversidade do Semiárido/MCTI (Processo: 558317/2009-0) pelo apoio financeiro concedido para realização das coletas em campo e pela bolsa concedida a AMSN.

\section{REFERÊNCIAS}

Brasil, 2002. Avaliação e ações prioritárias para a conservação da biodiversidade da Mata Atlântica e Campos Sulinos. In Biodiversidade brasileira: Avaliação e identificação de áreas e ações prioritárias para conservação, utilização sustentável e repartição dos benefícios da biodiversidade nos biomas brasileiros. Ministério do Meio Ambiente. Brasília, p. 215-266. Disponível em: <http://www.biodiversidade.rs.gov.br/arquivos/ BiodiversidadeBrasileira MMA.pdf $>$ [10/09/2011].

García Aldrete, A.N., 2006. Two new ptiloneurid genera (Psocoptera: Ptiloneuridae) from South America. Studies on Neotropical Fauna and Environment, 41: 133-137.
Garcia Aldrete, A.N. \& E.L. Mockford, 2009. A list of Psocoptera (Insecta : Psocodea) from Brazil. Revista Mexicana de Biodiversidad. 80: 666-673.

Lienhard, C. \& C.N. Smithers, 2002. Psocoptera (Insecta). World Catalogue and Bibliography. Instrumenta Biodiversitatis V. Muséum d'histoire naturelle. Genève, Suisse. 745p.

Mockford, E.L., 1993. North American Psocoptera(Insecta). Illinois, Sandhill Crane Press, 455p.

Smithers, C.N., 1990. Keys to the families and genera of Psocoptera (Arthropoda: Insecta). Technical Reports of the Australian Museum, 2: 1-82.

Valente, E.B. \& K.C. Pôrto, 2006. Hepáticas (Marchantiophyta) de um fragmento de Mata Atlântica na Serra da Jibóia, Município de Santa Teresinha, BA, Brasil. Acta Botanica Brasilica, 20: 433-411.

\section{Recebido em: 09/10/2011}

Aceito em: 16/10/2012

\section{Como citar este artigo:}

Silva-Neto, A.M., F. Bravo \& A.N. García Aldrete, 2013. Primeiro Registro de Ectopsocus titschacki Jentsch (Psocodea: Psocoptera: Ectopsocidae) para o Estado da Bahia: Uma Prova da Falta de Estudos nessa Ordem de Insecta no Brasil. EntomoBrasilis, 6(1): 86-88.

Acessível em: http://www.periodico.ebras.bio.br/ojs/index.php/ebras/article/view/198. doi:10.12741/ebrasilis.v6i1.198
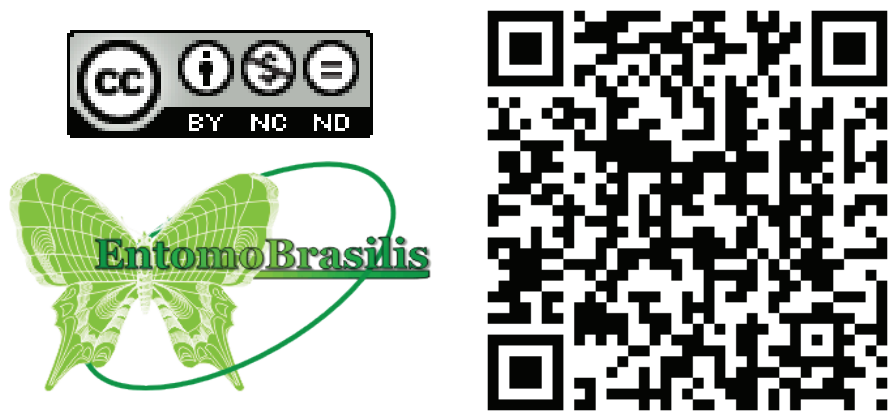